\title{
tic\&société
}

Vol. 9, No 1-2 | 1 er semestre 2015 - 2ème semestre 2015

Les religions au temps du numérique

\section{De la mobilisation de la communication numérique par les religions}

\section{David DOUYÈRE}

\section{(2) OpenEdition \\ 1 Journals}

\section{Electronic version}

URL: http://journals.openedition.org/ticetsociete/1822

DOI: 10.4000/ticetsociete.1822

\section{Publisher}

Association ARTIC

\section{Electronic reference}

David DOUYĖRE, « De la mobilisation de la communication numérique par les religions », tic\&société

[Online], Vol. 9, № 1-2। 1er semestre 2015 - 2ème semestre 2015, Online since 20 April 2019, connection on 01 May 2019. URL : http://journals.openedition.org/ticetsociete/1822 ; DOI : 10.4000/ ticetsociete.1822 
tic\&société - 9 (1-2), 2015

De la mobilisation de la communication numérique par les religions

David DOUYÈRE

Université François-Rabelais, IUT de Tours

Département Information-Communication

Équipe Prim

29, rue du Pont-Volant

37082 Tours cedex 2

david.douyere@univ-tours.fr 
De la mobilisation de la communication numérique

par les religions

\section{David DOUYÈRE}

\section{De la mobilisation de la communication numérique par les religions}

David DOUYĖRE est professeur de sciences de l'information et de la communication à l'institut universitaire de technologie de l'Université François-Rabelais à Tours, où il anime l'équipe de recherche Prim, "Pratiques et ressources de l'information et des médiations ". Ses recherches portent notamment sur la communication religieuse en contexte chrétien catholique: théorisations de la communication et théologie, communication imaginaire (dialogue mystique, communication angélique), prière, prédication, rôle des images, dispositifs numériques, concile Vatican II, ordre des frères prêcheurs (Dominicains). II co-anime le réseau de recherche Relicom, Communication et espaces du religieux. II a dirigé dans ce cadre, avec Stéphane Dufour et Odile Riondet, le numéro 38 (2014) de la revue MEl, Médiation et Information, « Religion et communication ».

Résumé : Tentant de constituer la liste des catégories d'emplois possibles du numérique en contexte religieux, cet article reprend quelques approches théoriques proposées de la question du numérique religieux et propose quelques ressources bibliographiques. II s'efforce enfin d'examiner les conditions d'étude de ces phénomènes.

Mots clés : numérique, TNIC, religion, communication.

Abstract: Concerning the use of digital communication by religious groups. The article suggests theoretical approaches to questions pertaining to religious uses of the digital field, it also provides bibliographical sources. This article also studies the ways in which digital techniques may be applied to religion.

Keywords: digital, ICT, religion, communication, Christianity, Islam, methodology.

\section{Resumen :}

De la movilización de la comunicación digital por las religiones. Buscando establecer la lista de las categorías de usos posibles de lo digital dentro de un contexto religioso, este artículo retoma ciertos enfoques teóricos sobre lo digital religioso y 


\section{David DOUYÈRE}

propone algunos recursos bibliográficos. Se insiste además en examinar las condiciones de estudio de dichos fenómenos.

Palabras clave: digital, TDIC, religión, comunicación.

Les religions, se formant de la communication et de la transmission de l'information, dogmatique et rituelle, ainsi que de la suggestion qu'elles opèrent de la présence du divin, ont utilisé l'ensemble des moyens d'information et de communication, dès leur naissance : ceux-ci ont contribué à construire un espace de signification socialement partagé et à accroître leur emprise, par le texte, le rite, le discours et l'image (ou son absence), suscitant ou mettant à distance l'affect, invitant à une communication avec des êtres non visibles, contribuant, par cette diffusion, à une régulation de l'espace social, fortement différencié et structuré.

Les technologies numériques de l'information et de la communication (TNIC) sont mobilisées aujourd'hui de la sorte par des groupes religieux, des mouvements spirituels et des sectes pour mettre en circulation des prières, transmettre des informations pour la pratique rituelle, diffuser une connaissance juridique, enseigner ou apprendre le rituel et le dogme, partager des interprétations, donner à voir des signes, des images ou des événements, et inviter à les rejoindre. Si elles ne forment pas nécessairement une « cybereligion » (Mayer, 2008 ; Cottin et Bazin, 2003), reliées qu'elles sont à des ensembles de pratiques, à des corpus législatifs et rituels, et à des institutions (Duteil-Ogata et al., 2015 b), à des lieux et des édifices, les TNIC produisent néanmoins un accompagnement infocommunicationnel de la pratique ou de la croyance religieuse dont certains fidèles, le plus souvent pratiquants, disposent. Ces dispositifs techniques accompagnent manifestement le développement ou la récession de certaines religions; elles en modifient parallèlement la visibilité et la représentation, les parant d'une modernité nécessaire auprès de certains publics.

Si les TNIC renouvellent l'apologétique et la propagande religieuses, au moins dans la forme, quand textes ou images de la tradition sont repris et rediffusés, parfois régénérés, sur ces supports, elles peuvent être les outils d'un nouveau prosélytisme, visant d'autres catégories de population. Ces technologies peuvent également être perçues comme transformant la relation que les religions entretiennent à leur « savoir » propre, autrefois difficilement accessible (comme par ex. dans le judaïsme; Stolow, 2014), au prix d'années d'études, et au «mystère » qu'elles annoncent ou décrivent et 
auquel elles introduisent (mystagogie, initiation...). En effet, les TNIC, publicisent, tandis que les religions constituent des espaces progressifs, normés et restreints permettant l'accès de seulement quelques-uns à l'information et à la connaissance juste du divin.

Nous proposons donc ici une revue succincte de quelques approches concernant la mobilisation des TNIC par les religions, avant de tenter de tracer la liste des usages et fonctions de ces dispositifs, puis de préciser quelques aspects des modalités d'étude de ces phénomènes.

\section{Des travaux sur TNIC et religions}

\subsection{Religions multiples dans l'espace numérique}

Les relations d'un certain nombre de courants religieux avec le numérique ont désormais été souvent étudiées de façon spécifique. Nous souhaiterions par conséquent indiquer ici brièvement quelques références, de façon non exhaustive afin de faciliter éventuellement les recherches (sans entrer toutefois spécifiquement dans ces travaux). Le judaîsme a été considéré dans son rapport aux TNIC notamment par H. Campbell (2010), par L. Podselver (2015), par Oren Golan, Lynn Schofield Clark et Jill Dierberg, Tsuriel Rashi (dans Campbell, 2013) mais aussi par Zénouda et Renucci (2014) et Golan (2011), tandis que M. Niculescu (2015) s'est intéressée à la médiation bouddhiste juive de la «pleine conscience ». Un certain nombre de travaux ont désormais été consacrés à l'islam et au numérique, par Gary Bunt (2009), Olivier Roy (2000), Abil Echchaibi (dans Campbell, 2013), K. R. Wheeler (2014), M. Lövheim (2015), C. Varlik (2015), C. Arminjon sur le droit chiite et internet (2015). La relation des Mourides du Sénégal au numérique a également été examinée (Bèye, 2011; Bondaz, 2015). H. Campbell (2010) a pour sa part mené une étude sur l'islam turc. Stefania Capone (1999) a consacré un article aux religions africaines sur internet. Le bouddhisme tibétain a été étudié notamment par Helland $(2014,2015)$ et Louise Connelly («Virtual Buddhism: Buddhist ritual in second Life », dans Campbell, 2013), ainsi que G. Grieve (2010). Erica Baffelli a consacré un article aux religions japonaises (in Campbell,

\footnotetext{
${ }^{1}$ L'auteur remercie l'équipe Prim (Pratiques et ressources de l'information et des médiations) de l'Université François-Rabelais de Tours pour les questions, les observations et remarques de ses membres lors d'une présentation préalable d'une version complète de ce travail au cours de son séminaire de recherche.
} 
2013), et F. Duteil-Ogata (2015) à certains rites funéraires japonais convoquant informatique et numérique. Heinz Scheifinger a proposé plusieurs articles sur l'hindouisme (2010, et dans Campbell, 2013), de même que Christopher Helland (2010). Enfin, le jaïnisme numérique a été étudié par $\mathrm{T}$. Vekemans (2014).

Nous ne reviendrons sur le christianisme, largement abordé dans cet article et étudié dans ce numéro de tic \& société, que pour citer quelques références complémentaires: une comparaison entre sites catholiques et protestants a été naguère proposée de façon pionnière par Corinne Abensour (2001), des analyses de liens entre les sites web catholiques ont été faites par Stéphane Amato et Eric Boutin (2014) ; Andrea Catellani a consacré trois études au site de spiritualité ignacienne (proche des jésuites) Notre-Dame du Web (2014 a et b, 2015) ; enfin Gustavo Gomez-Mejia (2014), auteur dans le présent numéro de tic \& société d'un article sur les powerpoints religieux, a consacré un article à la figure de sainte Rita, la «patronne des causes désespérées» sur Facebook. A. Filimon-Benea (2014) s'est intéressée aux liens de l'orthodoxie (roumaine) avec l'internet. P.-Y. Kirschleger (2015) a étudié le développement du site francophone protestant Jesus.net. Damien Mottier (2105) s'est intéressé à la mobilisation du numérique dans l'évangélisation et le culte au sein d'une église pentecôtiste charismatique. C. Vanel (2015) a travaillé sur la relation du mormonisme à l'internet et $A$. Julliard (2015) a mené des observations sur la fonction d'un site web anglican.

Céline Couchouron-Gurung (2007) a travaillé sur les Témoins de Jéhovah. On peut signaler également une étude, un peu en marge de ces approches spécifiquement religieuses, de la construction négociée de l'article de Wikipédia sur Jésus de Nazareth (Boutin, Amato, Putzka, 2014), plein d'enjeux religieux.

De façon plus transversale, deux ouvrages ont été notamment consacrés aux applications numériques dites de réseaux sociaux en lien avec les religions (Hope Cheong, Fischer-Nielsen, Gelfgren, Ess, 2012; Gillespie, Herbert, Greenhill, 2013). L'ouvrage collectif Le Religieux sur internet, dirigé par Fabienne Duteil-Ogata, Isabelle Jonveaux, Liliane Kuczynski et Sophie Nizard (2015 c) à l'issue d'un colloque de l'Association française des sciences sociales des religions organisé à Paris en 2013, permet d'enrichir de façon transversale ces approches grâce à de nombreuses analyses de l'investissement religieux du numérique en mobilisant des méthodologies et des cadres disciplinaires variés; quelques travaux qui en sont issus sont également disponibles dans 
Online - Heidelberg Journal of Religions on the Internet $\left(\mathrm{n}^{\circ} 8\right.$, 2015). Terminons en citant deux ouvrages généraux sur le numérique et le religieux (Karaflogka, 2006 ; Hadden et Cowan, 2000), de même que deux revues scientifiques en ligne spécifiquement consacrées à l'étude des religions dans leur communication numérique : le Journal of Religion, Media and Digital Culture ${ }^{2}$ et le Online - Heidelberg Journal of Religions on the Internet ${ }^{3}$. Les revues de sciences sociales des religions accueillent également des articles consacrés au numérique religieux. Mais laissons ces recensions bibliographiques à visée de repérage pour nous engager désormais dans la lecture de quelques approches du numérique religieux, en vue d'enrichir nos analyses, et de montrer la vitalité de ces travaux, et, quelquefois, leur caractère proprement heuristique.

\subsection{Approches croisées : quelques analyses}

Un certain nombre de travaux documentent en effet aujourd'hui l'approche de l'utilisation des TNIC en contexte religieux. Afin d'enrichir la réflexion sur les pratiques religieuses du numérique, nous donnons des éléments de synthèse de notre lecture de certains d'entre eux, qui permettent de mieux comprendre les pratiques religieuses du numérique. Évidemment, cela pourrait (et devra) être encore largement enrichi, tant les travaux, notamment nord-américains, sont nombreux.

La littérature étatsunienne (Helland, Campbell, Howard, notamment) semble s'être largement préoccupée du partage entre les pratiques purement numériques du religieux, qui en viendraient à le recomposer, et les pratiques numériques d'institutions, et plus largement de la question de la « communauté » formée ou accompagnée par ces dispositifs. S'est ainsi développée une réflexion sur la genèse numérique de communautés religieuses spécifiques. Les approches francophones (Jonveaux ; Bratosin, Tudor et Coman ; Douyère) nous semblent, outre cet aspect (Mayer, 2008), s'être plutôt intéressées à la dimension institutionnelle de l'utilisation des TNIC par le religieux, à la dimension innovante des dispositifs, voire, dans une perspective quasi-philosophique, à la dimension de subjectivité mobilisée. Cette approche n'est qu'indicative et n'a cependant pas de valeur taxinomique réelle. II n'y a pas lieu de la reprendre en tant que telle.

${ }^{2}<$ http://www.jrmdc.com/journal/index>, dernière consultation le 2 novembre 2015.

$3<$ http://journals.ub.uni-heidelberg.de/index.php/religions/index $>$, dernière consultation le 2 novembre 2015. 


\section{David DOUYÈRE}

Christopher Helland (2005) a proposé une célèbre distinction entre online religion et religion online, nuancée, distinguant une pratique religieuse qui serait vécue en ligne dans l'interaction d'une religion qui s'étendrait en ligne : la première proposerait médiations et espaces de prière, échanges, rituels en ligne, la seconde les horaires des offices et des informations concernant le culte, sur internet, opposant monde social vécu et information, les deux se croisant désormais sur un certain nombre de sites, reconnaît le chercheur.

Heidi A. Campbell suggère dans Exploring Religious Community Online (2005, rééd. 2010) d'étudier les pratiques religieuses numériques en considérant, dit-elle, les «identités narratives » (p.75 et suivantes) et les récits produits par ces communautés qui les portent. Des croyants échangent par courriel et forment ainsi discours et communauté. Elle rappelle que la communauté peut être perçue, en contexte chrétien, comme une représentation divine dans le monde (p.30), ou la représentation d'une relation intra-divine (la Trinité constituant le dieu chrétien) ; voire que la communauté peut désigner une relation humano-divine partagée (pp.30,153). Reprenant l'approche du juriste et théologien méthodiste Stanley Hauerwas (In Good Company: The Church as Polis, 1981), elle estime en effet qu'une église est une communauté formée par une histoire (story, p.39), établie sur un « récit de vérité » qui lui est propre. Campbell trace quelques éléments d'histoire des pratiques chrétiennes du numérique, depuis les forums Usenet des années 1980 jusqu'à la première église chrétienne en ligne, celle des Presbytériens du New Jersey, en 1992 (p.63), et audelà. Elle évoque les conceptions de la technologie comme projet de Dieu, et visant à rendre semblable à Dieu (théurgie, p.57). Elle rappelle qu'internet a été perçu comme Seconde Réformation (Réforme), en s'appuyant sur l'analogie classique des discours d'escorte des TNIC avec l'imprimerie, souvent valorisée par les spécialistes en s'appuyant sur les travaux d'Elizabeth Eisenstein. Son étude se consacre spécifiquement à l'étude de trois communautés religieuses en ligne, The Community of Prophecy, The Online Church, et The Anglican Communion on line (p.80 sq) ; ces communautés forment des "réseaux spirituels», par une dimension langagière et collective. Des symboles, de l'affection (cyberhugs, p.217) sont également partagés; la prière en ligne constitue un lien commun. En s'appuyant sur les modèles ecclésiaux définis par le théologien jésuite Avery Dulles en 1974 (Models of the Church) elle distingue (pp.154-156) une communauté prophétique (se vivant dans l'Esprit saint), une communauté de hérauts et serviteurs (axée sur la proclamation, l'étude et le service), et une communauté sacramentelle (symbolique). Elle relève, avec Dulles, la fonction « assemblatrice », nous dirions 
unifiante, du langage (p.34). En ligne, dans ces communautés, apparaissent relations, soin (care), valeur, connexion, communication intime, foi partagée, dans un réseau relationnel qui est aussi un réseau narratif (p.189).

Dans When Religion Meets New Media (2010), Campbell élargit son approche au judaïsme, étudié en Israël, notamment dans les communautés orthodoxes, et à l'Islam turc. Elle donne à sa recherche une nouvelle assise théorique, celle de la mise en forme socio-religieuse de la technologie (religious-social shaping of technology), qui suppose d'examiner la tradition historique, les valeurs contemporaines et objectifs d'une religion, la façon dont l'innovation technologique se trouve négociée et les discours justificatifs de l'appropriation. Étudiant le judaïsme orthodoxe, elle montre la résistance à la technologie, et les tentatives de "cachérisation » de l'internet (p.117). Pour l'Islam, elle montre le souci de créer une " umma digitale ", et les tentatives de centralisation, comme en 1997 avec le site qatari Islam online. L'islam tend à faciliter la pratique religieuse par la technique (p.127), notamment par le calcul des horaires de prière suivant la localité de l'orant, des alertes de prière, ou la recherche de la direction de La Mecque. Ces dispositifs permettent aussi l'étude du Coran. Ainsi s'établit selon la chercheuse le façonnage socio-religieux de la technologie. Campbell estime au passage (p.130) que ces technologies permettent de séduire une jeunesse connectée cherchant à négocier identité culturelle et religieuse, et que la technique permet également de remettre en forme des rituels religieux traditionnels (p.131). Un travail de labellisation et de prescription des usages s'opère dans les mouvements religieux (p.136). Dans le courant de l'« e-vangélisme » (évangélisme numérique), par exemple, apparaît un mandat religieux d'évangélisation qui requiert l'utilisation de ces outils. Un cadrage organisationnel de l'internet pointe souvent. Parfois, ces techniques permettent l'émergence d'un mouvement de contre-culture religieuse (mouvement protestant dit de l'Église émergente, Emerging Church, p.150), dans la tradition des Jesus People des années 1960. La chercheuse étudie également la régulation progressive du téléphone portable dans le judaïsme (p.162). H. Campbell suggère aussi des pistes de recherche (p.190) : analyse des usagers et de leur expérience, étude des pratiques notamment dans les religions asiatiques. Pour Campbell (2015), une nouvelle forme de « religion en réseau ", ou religion réticulaire, apparaît, s'établissant sur un réseau d'interactions plus que sur des communautés instituées. 


\section{David DOUYÈRE}

Dans Digital Jesus ${ }^{4}$, Robert Glenn Howard (2011) pose précisément l'existence, à l'issue d'une longue recherche menée sur les échanges en ligne, d'une communauté chrétienne fondamentaliste, trouvant dans la Bible les clés d'interprétation du monde contemporain et de son avenir ( End Times »: la fin des temps, ou les Derniers Jours). II qualifie cette communauté de «virtual ekklesia », ekklesia virtuelle (virtuelle signifiant ici : manifesté par ses effets), faisant par ce terme référence au rassemblement des premières communautés chrétiennes, telles que les Actes des apôtres ou les écrits de Paul de Tarse permettent de les comprendre. Ce terme lui permet de distinguer cette communauté d'échange d'une moderne "Église » instituée. Une communauté d'échange se forme dans la correspondance numérique à partir de, notamment, l'interprétation « biblique » des événements du 11 septembre 2001, que certains fondamentalistes tentent d'interpréter à la lumière de la Bible, en les voyant comme des signes. Se forme ainsi une communauté d'interprétation, dans l'échange. Une des contributrices de ce réseau, Marilyn Agee, par son site web Bible Prophecy Corner, reprend et nourrit ces discussions, menées souvent par courriel, des interprétations que Dieu lui-même lui révèle (ce qui est fort utile au débat). Howard étudie les réseaux formés par ces échanges entre fondamentalistes depuis les newsgroups suivis en 1992. II observe qu'une communauté d'interprétation participative et «prophétique » se compose alors. Elle constitue une forme religieuse à part entière, qui n'a d'existence que dans la représentation que ses membres ont qu'elle existe (p.13), en tant que «communauté imaginée », et dans leurs échanges. Elle n'a en revanche pas de modalité institutionnelle séparée. Elle transcende les sources traditionnelles d'autorité (animateurs et institutions) et est animée, estime-t-il, d'une " tolérance » à l'égard des opinions énoncées en son sein. Cette «digital ekklesia » est donc "vernaculaire », pour Howard et forme, comme une institution ecclésiale chrétienne, le corps de Jésus, mais sous forme numérique, d'où le titre de son ouvrage.

Quelques approches s'intéressent par ailleurs - sans lien avec le numérique, mais elles sont utiles au chercheur qui entend considérer une perspective historique plus ample de la sollicitation religieuse des techniques - plus largement à l'histoire des techniques mobilisées par le religieux. Jeremy Stolow propose ainsi implicitement dans Deus in Machina: Religion, Technology, and the Things in Between (2012) de placer le développement numérique du religieux dans un

\footnotetext{
${ }^{4}$ L'auteur remercie Yves Jeanneret et Véronique Richard d'avoir attiré son attention sur cette recherche.
} 
continuum des rapports des religions à la technique. En France, l'historien Michel Lagrée avait proposé dans La Bénédiction de Prométhée (2000) une étude des rapports de l'Église catholique aux techniques de communication.

Dans quelques articles consacrés aux powerpoints de prière et aux images numériques chrétiennes de piété (Douyère, 2015, 2014 b, 2011), nous nous sommes intéressé pour notre part aux transpositions digitales de l'image de piété et de médiation chrétienne catholique, en montrant comment, à partir de l'étude de diaporamas de prière réalisés par une communauté bénédictine québécoise, une dimension pédagogique, méditative et « mystagogique » (d'introduction au "mystère chrétien ») était incarnée par ces dispositifs de «prière assistée par ordinateur », qui convoquent la présence de Jésus, pour le faire exister par l'invocation de son nom. Sorte de «joli missel numérique », comme le disent les conceptrices, ces dispositifs reproduisent l'appareillage matériel dont Michel de Certeau avait relevé dans La Faiblesse de croire la présence autour du croyant. Ils sont issus en l'occurrence d'un «artisanat monastique numérique », inscrivant la production d'images numériques dans la continuité d'une production d'image peintes ou collées sur bois dans les monastères, par exemple. Dans le présent numéro de tic \& société, Gustavo Gomez-Mejia prolonge ce travail par une étude sémiotique de chaînes de powerpoints latino-américains.

Dans leur singulier article «La pratique du sacré dans le World Wide Web: une expérience innovante de la norme ", Stefan Bratosin, Mihaela Tudor et lacob Coman (2010) insistent également sur l'innovation normative qui consiste dans l'utilisation de dispositifs numériques en contexte religieux: « toute pratique du sacré dans le cyberespace est l'expérience innovante d'une norme » (p.5). Déplacement et creusement des représentations, le sacré en ligne est l'espace du croyant indifférencié. La «formule » y acquiert un sens de reconnaissance: "L'appel contenu en cette formule importe peu. II importe seulement qu'elle soit perçue comme claire et visible de loin » (p.10), car les formules "apparaissent aussi douées d'une énergie motrice » (Idem). Les auteurs s'interrogent également sur la légitimation du dispositif technique par le sacré, «condition soupçonnée de pouvoirs magiques » (p.12). La technique se fait alors médiation : "l'invisible a priori infiniment grand et absolument autre, et le tout petit pratiquant, sont pris ensemble en tension technologique. Dans ce contexte, l'invisible n'est rien que l'autre extrémité de la monstrueuse technologie, où l'on peut tout glisser parce qu'elle tient tout. » (p.13). Compte surtout l'arc qui tend l'un et l'autre, aux deux bouts, du sujet et du divin. 
La mise en visibilité des grandes figures des pratiquants du sacré mobilisables permet notamment au pratiquant d'« emporter » sa foi. La pratique du sacré en ligne attire l'internaute vers autre chose et provoque, à chaque consultation de site, "aversion vis-à-vis de l'actuel et attachement au possible, notamment d'une expression directe sans représentant intercalé » (p.23). Cependant, "Le subjectivisme du pratiquant du sacré n'a qu'un but topographique ». (p.26) : il s'énonce d'un lieu. En effet, «Dans la pratique internaute du sacré, une personne ne produit pas du sens parce qu'elle veut montrer son essence, mais parce qu'elle souhaite seulement se révéler à elle-même qu'elle est quelque part » (Idem). "[J]usqu'à ce qu'il sente lui-même qu'il est là, partie constitutive de la représentation de son injonction de la règle de foi » (Idem), l'internaute a recours à ce dispositif. Cette approche philosophique et générique (un sujet transcendantal de l'expérience du web du sacré, générique) d'ego-localisation permet de poser l'attente d'un internaute face à des sites religieux. "Il expose sa foi comme une affiche, où il déchiffre qu'ici, il est chez lui. » (p.28) L'approche est ici plus existentielle et symbolique que pratique : elle questionne le recours au numérique sacré, en termes anthropologiques. Les questions qu'elle pose pourraient être également posées à la pratique religieuse " analogique ». Stefan Bratosin et Mihaela Tudor (2014) s'efforcent par ailleurs d'analyser, plus largement, avec d'autres chercheurs, les modes de communication du religieux (dénommés «la foi », le sacré, le symbolique) dans l'espace public.

Isabelle Jonveaux étudie dans Dieu en ligne (2013) et dans quelques articles (Jonveaux, 2007, 2009, 2015) les pratiques monastiques catholiques de connexion à l'internet et montre comment le "retrait du monde " s'accommode de connexions à l'internet, notamment pour les jeunes entrants (les novices et les étudiants), par une étude portant principalement sur la France, l'Italie et l'Autriche. Une clôture est-elle encore telle quand le monde y pénètre par la communication numérique ? Cependant, internet permet également de demeurer à l'intérieur, notamment par achats et prise d'information en ligne. II peut faciliter les dons aux communautés monastiques. Les moines semblent davantage connectés que les moniales, ainsi que les plus jeunes. Tout dépend en réalité de la façon dont la communauté monastique entretient des liens soutenus, économiques (vente de produits monastiques, tourisme....), mais aussi pastoraux et de service, avec le monde. Internet paraît aussi, suivant la ligne pontificale (messages pour les communications sociales), constituer un vecteur d'évangélisation touchant les gens « là où ils sont, dans leur vie quotidienne »(p.57). Jonveaux montre comment les religieux 
se mettent en scène en ligne, y compris sur Facebook, figurant parfois l'épanouissement et la joie, de façon attractive, visant de la sorte à récuser certaines perceptions de la vie religieuse. Parfois s'y livrent, par des photos, des éléments de vie personnelle et de valorisation de soi.

Jonveaux s'intéresse également au «jeûne numérique » (pp.89, 224 et suivantes) pratiqué par certains religieux qui se consiste à se priver certains jours d'internet ou de consultation des applications de réseaux sociaux ; elle poursuit actuellement une recherche sur ce point. Ainsi, « [...] vivre sa religion peut désormais se faire sur la toile : [...] l'Internet semble proposer une panoplie complète de pratique religieuse. » (p.13). Elle forme enfin l'hypothèse, avec les sites de prière et les « retraites» proposées en ligne (par le site web et les applications d' "Une retraite dans la ville ») d'une " religion à domicile » (pp.184, 205), peut-être expliquée aussi par la paresse (!), mais surtout par la réduction des effectifs de clercs en Europe (souligné également par Jean-Pierre Bacot, 2013) et par le dé-tissage d'un maillage territorial paroissial en résultant. II pourrait ainsi constituer un «nouvel agent de proximité de la religion » (p.231). Pour la sociologue, l'individualisation, le "bricolage » et l'exculturation (selon l'expression célèbre de Danièle Hervieu-Léger) du christianisme expliquent également en partie ce mouvement du numérique religieux, qui permet toutefois un affichage discret de ses pratiques religieuses. Le numérique religieux pourrait aussi constituer un « ersatz virtuel » (p.184) de la pratique religieuse ou une «présence religieuse de substitution » (p.186). Elle relève aussi que «la pratique sur Internet vient reposer la question de la médiation dans la relation religieuse » (p.216). Ailleurs, Jonveaux (2015) s'interroge sur la présence du virtuel, comme catégorie de pensée, au sein même de la religion chrétienne.

\section{Des mobilisations informationnelles et communicationnelles variées}

L'extension est très vaste des dispositifs numériques mobilisés par les religions. Nous proposons de parcourir d'abord de façon générique les champs et usages possibles, repérés, des TNIC par le religieux, afin d'ouvrir, par une sorte de carte inachevée, des territoires de recherche, mais aussi d'apprendre à les interconnecter.

Les réseaux numériques sont aujourd'hui mobilisés par différents acteurs religieux, nous semble-t-il, dans plusieurs perspectives : 


\section{David DOUYÈRE}

- l'actualisation de l'information rituelle, concernant par ex. les heures d'allumage des bougies (judaïsme), les heures de prière ou le début d'un jeûne rituel (islam) ; religieux

- faire connaître les heures et lieux des différents services

- informer de la vie d'une communauté religieuse, par ex. paroissiale ou régionale (diocésaine) ;

- permettre de recevoir les conseils d'un ministre religieux (islam, judaïsme, etc.) ;

- diffuser les textes de prédications, de commentaires de la tradition (exposés bouddhistes, homélies catholiques, prédications protestantes) ou des vidéos de ces herméneutiques religieuses publiques ;

— diffuser des images de piété numérisées ou des créations visuelles de piété numériques ;

- effectuer un partage de prière ou d'intentions de prière en ligne (travaux d'Andrea Catellani, 2014 a et b, d'Isabelle Jonveaux, 2007, 2013) ;

— proposer des ressources de prière en ligne ;

- proposer la cueillette d'une citation biblique ou spirituelle suivant un algorithme, la pertinence du choix étant ensuite éventuellement attribuée par le récepteur à l'Esprit saint visant personnellement le requérant ;

- le partage ou la reprise de documents d'animation de groupe, mais aussi de textes proposant des orientations spirituelles ou pastorales ;

- le partage de textes anciens, et commentaires (judaïsme, patristique, Islam) ;

- aider au recrutement d'une communauté (communauté Saint-Martin, par ex.) ;

- faire émerger des positions pour faire pression sur la hiérarchie et l'institution, par ex. ecclésiale catholique, pour la condamnation ou l'abandon de certaines pratiques, ou lois (blogueurs catholiques, par ex.), ou diffuser une parole religieuse dans l'espace public (cf. Josselin Tricou, dans ce numéro de tic \& société) ;

- susciter et fédérer une parole de résistance face à l'institution et d'émancipation à l'égard de la dogmatique ou de l'ecclésiologie officielle (CCBF, Conférence catholique des baptisé-e-s francophones: «Ni partir, ni se taire ») - soit la constitution d'une opinion publique interne, d'un espace public interne à l'Église catholique ; 
- inviter à une pratique adaptée à la modernité, par des tutoriels religieux, dans le judaîsme et l'islam (cf. Lucie Le Guern Formenti, dans ce numéro de tic \& société ; Bunt, 2009 ; Campbell, 2010) ;

- recueillir des bonnes volontés en vue d'une action sociale à caractère religieux, fédérer des ressources pour un projet ;

- inviter au don (Jonveaux, 2013), vendre des livres ou des images religieuses en ligne, voire des prestations sacramentelles ou de piété (cierge numérique, faire dire une messe) ;

permettre des rencontres amoureuses intra-
confessionnelles
rencontresprotestants.fr. etc.)
- propotoks,
défunts;
$\quad$ - entrer en relation avec des puissances célestes par le
biais d'internet (altération de pages ou de messages, etc.).

Ces actions et ces services peuvent évidemment se combiner. II nous paraît néanmoins délicat de construire une typologie des usages (toujours insatisfaisante), tant la diversité des pratiques nous paraît grande. S'il fallait en proposer une, nous distinguerions neuf catégories : pratiques orantes (prière), pratiques légalistes (informations sur la loi religieuse qu'il convient d'appliquer), pratiques de socialisation religieuse (échanges entre fidèles, ou avec des clercs), pratiques de connaissance (acquisition ou requête d'un savoir religieux), pratiques visuelles et discursives (diffusion d'images et de discours alimentant la piété), guidage de l'action religieuse (conformation aux règles de piété dans le cadre de la vie sociale), pratiques informationnelles (sur l'exercice du culte), pratiques politiques (influence dans l'organisation religieuse), incitation à l'action (rejoindre une communauté, faire un don). L'expression divine par le biais de ces technologies nous paraît un cas à part, qui relève davantage de l'interprétation individuelle que de la prescription religieuse.

Ces actions et services, bien qu'ils tendent à se multiplier sont toutefois très peu nombreux comparés à ceux qu'assurent des institutions ou communautés religieuses inscrites dans des territoires et opérant un service religieux présentiel. On remarque toutefois que s'opère une relative - mais partielle numérisation du service religieux auprès du public qui accède à ces ressources. 


\section{David DOUYÈRE}

Les TNIC s'inscrivent donc dans la vie informationnelle et communicationnelle des religions, qu'elles modifient en partie. Une première dimension est sans doute la dimension territoriale. Lorsqu'une religion s'appuie sur un maillage territorial, le réseau numérique peut venir transformer le rapport à celui-ci, voire l'outrepasser. Échanger avec un imam pakistanais ne requiert plus un long voyage initiatique, interroger un talmudiste israélien peut se faire par le web. Les dispositifs numériques offrent donc le choix de la consultation et du recours. Une seconde dimension est temporelle: sont potentiellement rendus accessibles en quelques instants des " savoirs » qui pouvaient demander des années d'acquisition (étude islamique, juive, bouddhiste, ou même chrétienne), la mise en relation d'adeptes est facilitée, tout comme la coordination d'événements. Si le religieux numérique permet donc une accélération, il propose aussi des espaces de ralentissement, s'offrant parfois comme une alternative à l'accélération effrénée des sociétés de la modernité tardive, puisqu'une religion, c'est aussi une offre de temporalité (inscription dans l'histoire et une tradition, un temps théologique et eschatologique, une célébration rituelle temporellement structurée, à la fois progressive et cyclique, ou l'instant).

La question majeure est de savoir si la numérisation partielle de l'information et de la communication religieuse conduit ou non à une désintermédiation en matière religieuse. II nous semble le plus souvent s'opérer non pas tant une disparition ou une réduction des intermédiaires, qui n'est qu'apparente, qu'une réintermédiation, soit que des clercs offrent leurs services et contenus en ligne, soit que des sites web et des applications assurent cette fonction. Surtout, des plateformes et bases de ressources (comme Youtube, ou Facebook) et des moteurs de recherche assurent désormais cette intermédiation (de l'intermédiation), ne serait-ce que par leur dimension architextuelle et structurante à l'égard des contenus. On assiste donc certainement davantage à un déplacement de l'intermédiation religieuse, qu'à sa suppression. De nouveaux acteurs, laïcs, assurent certainement cette fonction, quand les institutions religieuses n'investissent pas d'elles-mêmes cette dimension. Sans doute l'enjeu pour ces dernières est-il de ne pas perdre le contrôle de cette intermédiation.

\section{Modalités d'étude}

La difficulté de l'étude des enjeux religieux de la communication numérique, ou plutôt de la façon dont les religions recourent aux $\mathrm{TNIC}$, relève notamment du croisement 
d'une diversité de champs concernés par cette question. II semble d'une part difficile de séparer les phénomènes religieux (par ex. en ligne) des phénomènes culturels (Smith, 2014) ; faut-il même les nommer de façon spécifique? Certains auteurs ont fait le choix de parler de "sacré » (Dufour, 2011 a et b; Dufour et Boutaud, 2013) pour privilégier une approche anthropologique, et transversale (Douyère, 2014 a). Le religieux peut ensuite, nous semble-t-il, rarement être pensé sans lien avec le politique (Lambert, 2014 ; Gonzalez, 2013). II appelle, enfin, une compréhension de ses enjeux propres, théologiques et « spirituels ", comme disent certains de ses acteurs, soit des modes de réalité que créent ses discours et figures.

Le théisme méthodologique (Piette, 2003) nous semble également une posture nécessaire en ce qu'au lieu de poser un scepticisme qui refuserait de considérer la vérité possible de l'objet de la croyance, elle accepte un temps la possibilité de la tenir pour vraie, le temps de l'étude. Admettre qu'il soit possible de considérer que croire est une opération critique (Claverie, 2003), est une façon de laisser venir la forme croyante afin de l'étudier, de saisir le «monde » possible du religieux, sans le réduire ou l'ignorer d'une opération critique externe.

II nous paraît également important, avant tout, de ne pas s'en tenir à une étude du religieux en ligne, mais de considérer ces pratiques sur l'horizon des pratiques religieuses dans leur ensemble. Certes, l'étude de corpus textuels et de discours, d'images religieuses en ligne est commode, la " présentation " est faite par les acteurs, mais ceci n'indique le plus souvent qu'une petite partie des pratiques, pensées et discours des acteurs. On peut voir pourtant des actes d'un colloque consacré au religieux ne quasiment jamais aborder les pratiques et discours des personnes confessant une religion. Une communauté religieuse se présente-t-elle en ligne ? Mais que dit-elle par ailleurs à ses novices, quelles sont les règles morales réelles pratiquées dans la communauté (Jonveaux, 2013), comment s'y pratiquent les connexions à l'internet ? II y a un risque de virtualiser la recherche sur le religieux, sauf en quelques cas, à l'éloigner des corpus (rituels, ouvrages théologiques, objets et matériels de piété, etc.), des pratiques et des situations où le religieux se trouve mis en œuvre. Peutêtre ce type d'étude permet-il à la fois une recherche rapide, et surtout de « tenir le religieux à distance ". Peut-être est-il moins éprouvant pour le chercheur (dont on tentera peut-être d'évaluer l'adhésion au mouvement religieux d'une façon qui lui paraîtra moins pesante, ou qui ne fera pas l'objet d'une démarche de prosélytisme ?) qu'une étude de terrain, qui l'expose physiquement et mentalement (être cognitivement occupé par cet objet religieux et le porter dans l'esprit) à la 


\section{David DOUYÈRE}

prégnance religieuse («Vous êtes chrétien?»). Une telle position, qui considèrerait seulement des énoncés numériques, et se tiendrait à distance du terrain, risque aussi de conduire à écarter le chercheur de la prétention religieuse (une théorie explicative du monde) et de sa portée. II faut sans doute accepter d'être quelque peu pénétré par le religieux pour l'étudier, et de se saisir de ses pratiques, de ses lieux et discours, des récits qui en sont formés, et qui ne peuvent être, le plus souvent, seulement numériques.

L'étude proprement dite de dispositifs numériques religieux nous semble enfin devoir être replacée dans le contexte des pratiques et de l'histoire du mouvement étudié. La rencontre des pratiquants ou des confessants, de personnes qui s'inscrivent culturellement dans ces pratiques, nous apparaît nécessaire. Prendre au sérieux les modes d'existence (Latour, 2012) posés par les agents nous semble important, puisqu'il s'agit parfois de réalités immatérielles (si l'on peut dire). Regarder et considérer les espaces physiques de rassemblement ou de culte (Salatko, 2014), considérer les temporalités spécifiques de la vie « spirituelle », observante ou religieuse étudiée. Sans doute convient-il à la fois de considérer les objets numériques, en s'intéressant à leur contenu propre (facilement oublié, nous y reviendrons), leur forme visuelle, graphique et dynamique, mais aussi à leurs producteurs et conditions de production, voire, si cela est possible, aux pratiques qui se les approprient ou non (Hoover, 2006). II nous semblerait gênant de s'en tenir au "texte présenté en ligne » ou de croire que s'opère ce qui est inscrit.

Le numérique est - et peut-être est avant tout - un espace discursif et iconique d'argumentation, d'influence et d'incitation, dans lequel les courants religieux vont pouvoir s'exprimer et affirmer leur interprétation propre, et tenter d'infléchir le discours ou les pratiques d'une institution religieuse, ou d'un pays. Le mouvement socio-religieux «La Manif pour tous» s'est ainsi fortement servi du web, en France, dans la lutte contre la loi ouvrant le mariage aux couples de personnes de même sexe (2013), dite loi Taubira, pour mobiliser contre cette loi et diffuser une forte réticence à l'égard du mariage de couples homosexuels. 


\section{Conclusion}

Étudier la mobilisation du numérique par les religions, c'est donc étudier l'extension de la médiatisation du religieux, sa mobilisation de l'ensemble des médiations communicationnelles, sa plasticité communicationnelle. Les TNIC font partie de ces dispositifs et se trouvent présentes sur d'autres supports, qu'elles viennent compléter, dans une perspective qualifiée de transmédia. Elles remplissent des fonctions info-communicationnelles très variées, de l'information sur un office ou l'heure d'un rituel à la pratique de la " prière " et à l'échange sur le sens des rituels. Sans doute sont-elles à étudier de façon non séparée, mais comme s'insérant dans un continuum de pratiques (ou non), et en lien avec d'autres pratiques sociales (préparation de la nourriture, toilette intime, vêture, pratiques sexuelles, reproduction, relations genrées, repos et activité, pratiques mortuaires et de deuil, aide aux pauvres et aux malades, paramétrés par le religieux...), et au "sens » prêté au religieux par les agents, non sans se saisir de la dimension politique que forme l'ensemble de ces pratiques, ou que dessine spécifiquement la prétention d'une confession religieuse. Sans survaloriser le sens, ni le minoriser. Puisque le religieux est fabrique et diffusion de sens, très proche en cela de la communication. Machine à sens, le religieux s'empare en effet de tous les dispositifs matériels possibles pour figurer la signification de l'absent (ou le manque), et le message qu'il nous lègue, et former une communion référente, dans une perspective de structuration sociale, culturelle et politique. Communication par excellence, puisqu'il n'est même pas besoin que l'immatériel parfois référé parle. On s'en occupe. Communication par excellence, qui dispense même l'immatériel de l'existence, puisqu'elle lui en confère une, par le langage et l'image, pratiqués à plusieurs. ${ }^{5}$

\footnotetext{
${ }^{5}$ Cet article est dédié à la mémoire de Hodda Ben Khalifa Saadi, Hyacinthe Koma et Romain Didier, victimes des attentats de Paris le 13 novembre 2015, serveurs au café où une partie de ce travail a été préparée.
} 


\section{Références bibliographiques}

ABENSOUR C. (2001), « Les modalités paradoxales de la présence chrétienne sur internet », dans SFSIC, Émergence et continuité dans les recherches en information et communication, Actes du $12^{e}$ congrès national de la Sfsic, Paris, Société française des sciences de l'information et de la communication, pp.105-110.

AMATO S., E. BOUTIN, 2014, «De l'autoréférentialité de l'Église catholique dans les environnements numériques », dans S. BRATOSIN et M. A. TUDOR (dir), 2014, Espace public et communication de la foi, Actes du $2^{\mathrm{e}}$ colloque international ComSymbol les 2-3 juillet 2014, Béziers, France, larsicEssachess, s.l. [Les Arcs-sur-Argens], larsic, pp.317-328.

ARMINJON C., 2015, « La diffusion d'internet et l'ouverture d'un nouveau champ dans le droit islamique chiite ", dans F. DUTEIL-OGATA, I. JONVEAUX, L. KUCZYNSKI et S. NIZARD (dir), Le religieux sur internet, Paris, AFSR, L'Harmattan, pp.249-259.

BACOT J.-P., 2013, Une Europe sans religion dans un monde religieux, Paris, Le Cerf.

BELLAR W., H. A. CAMPBELL, K. J. CHO, A. TERRY, R. TSURIA, A. YADLIN-SEGAL et J. ZIEMER, 2013, « Reading Religion in Internet Memes », Journal of Religion, Media and Digital Culture, vol.2, $\mathrm{n} \%$, <http://jrmdc.com>, dernière consultation le 2 novembre 2015.

BERNARD A., 2013, “Le pèlerinage aujourd'hui : entre socialité et hagiothérapie », Questions de communication, $\mathrm{n}{ }^{\circ 23}$, pp.57-78.

BÉYE C., 2011, « Les mourides du Sénégal face aux défis des nouvelles technologies, le cas d'internet ", Le temps des médias, $\mathrm{n}^{\circ} 17$, pp.111-117.

BOILLAT J.-C., F.-X. AMHERDT, 2013, Web \& Co et pastorale, les NTIC et la transmission de la foi, Saint-Maurice (Suisse), Saint-Augustin, «Perspectives pastorales », $n^{\circ} 6$.

BONDAZ J., 2015, «Images cultuelles et écrans mourides, deux cas de controverse iconographique et religieuse sur internet », dans F. DUTEIL-OGATA, I. JONVEAUX, L. KUCZYNSKI et S. NIZARD (dir), Le religieux sur internet, Paris, AFSR, L'Harmattan, pp.261-274.

BOUTIN E., S. AMATO, M. B. PUTZKA, 2014, “"Jésus de Nazareth sous Wikipédia": analyse de la dynamique contributive et collaborative ", dans S. BRATOSIN et M. A. 
TUDOR (dir), 2014, Espace public et communication de la foi, s.l. [Les Arcs-sur-Argens], larsic, pp.343-352.

BRATOSIN S. et M. A. TUDOR (dir), 2014, Espace public et communication de la foi, Actes du $2 e$ colloque international ComSymbol les 2-3 juillet 2014, Béziers, France, larsicEssachess, s.I. [Les Arcs-sur-Argens], Iarsic.

BRATOSIN S., M.A. TUDOR, I. COMAN, 2010, « La pratique du sacré dans le world wide web: une expérience innovante de la norme ", Sciences de la société, n81, pp.121-134, $<$ http://sds.revues.org/875> , dernière consultation le 26 octobre 2015.

BUNT G., 2009, iMuslims, Rewiring the House of Islam, Chapel Hill, The University of North Carolina Press.

CAMPBELL H., 2015, " Les relations entre religion en ligne et hors ligne dans une société en réseaux », trad. C. BirkanBertz, dans F. DUTEIL-OGATA, I. JONVEAUX, L. KUCZYNSKI et S. NIZARD (dir), Le religieux sur internet, Paris, AFSR, L'Harmattan, pp.95-124.

CAMPBELL H. A. (dir), 2013, Digital Religion, Understanding Religious Practice in New Media Worlds, New York, Routledge.

CAMPBELL H. A., 2010, When Religion Meets New Media, New York, Routledge.

CAMPBELL H. A., 2005, Exploring Religious Community Online, We are One in the Network, New York, Peter Lang [2010].

CAPONE S., 1999, «Les dieux sur le Net. L'essor des religions d'origine africaine aux États-Unis », L'Homme, vol.39, pp.47-74.

CATELLANI A., 2015, "Pastorale et prière en ligne : le cas du site Notre Dame $d u$ Web ", dans F. DUTEIL-OGATA, I. JONVEAUX, L. KUCZYNSKI et S. NIZARD (dir), Le religieux sur internet, Paris, AFSR, L'Harmattan, pp.203-216.

CATELLANI A., 2014 a, «Images électroniques pour la prière: sémiotique et archéologie du site 'Notre Dame du Web' ", dans F. LAMBERT (dir), Prières et Propagandes. Etudes sur la prière dans les arènes publiques. Suivi du livre I de La Prière de Marcel Mauss, Paris, Hermann, pp.331-346.

CATELLANI A., 2014 b, «Prier en ligne à partir d'images : observations sémiotiques sur le site 'Notre Dame du Web' », MEl, Médiation \& Information, $\mathrm{n}{ }^{\circ 38}$, pp.101-112.

COTTIN J., J.-N. BAZIN, 2003, Vers un christianisme virtuel ? Enjeux et défis d'Internet, Genève, Labor et Fides. 
CLAVERIE E., 2003, Les guerres de la Vierge. Une anthropologie des apparitions, Paris, Gallimard.

COUCHOURON-GURUNG C., 2007, "Les Témoins de Jéhovah sur Internet, L'utilisation du web dans la mobilisation des acteurs d'une controverse ", Archives de sciences sociales des religions, $\mathrm{n}^{\circ} 139$, pp.139-156, $<$ https://assr.revues.org/9433>, dernière consultation le 2 novembre 2015.

DOUYERE D., 2015, «Accompagner et susciter la prière à distance : "les prières méditatives en diaporamas" de l'abbaye bénédictine Sainte-Marie des Deux-Montagnes ", dans F. DUTEIL-OGATA, I. JONVEAUX, L. KUCZYNSKI, S. NIZARD (dir), Le Religieux sur internet, Paris, AFSR, L'Harmattan, pp.217-230.

DOUYERE D., 2014 a, "La recherche en Sic sur le sacré et le religieux ", Les Cahiers de la Sfsic (Société française des sciences de linformation et de la communication), $\mathrm{n}^{\circ} \mathrm{g}$, janvier, pp.107-116.

DOUYERE D., 2014 b, «L'image de piété chrétienne, objetsupport de la croyance ? Communiquer la foi par l'image, de l'imprimé au numérique ", Recherches en communication, n ำ, pp.29-46.

DOUYERE D., 2011, «La prière assistée par ordinateur », Médium, n²7, pp.140-154.

DRESCHER E., K. ANDERSON, 2012, Click 2 Save: The Digital Ministry Bible, New York, Morehouse Publishing.

DUFOUR S., 2011 a, " Les nouvelles figures du sacré. Ou la circulation culturelle d'une notion sensible ", dans P. LARDELLIER (dir), La métamorphose des cultures. Sociétés et organisations à l'ère de la globalisation, Dijon, éditions universitaires de Dijon, pp.181-192.

DUFOUR S., 2011 b, « Les marques sont-elles des reliques postmodernes? Regard sur les nouveaux objets sacrés", Essachess, Journal for Communication Studies, vol.4, n²(8), pp.93-103,

$<$ http://www.essachess.com/index.php/jcs/article/view/129>, dernière consultation le 2 novembre 2015.

DUFOUR S., J.-J. BOUTAUD, 2013, «Extension du domaine du sacré ", Questions de communication, n²3, pp.730.

DUTEIL-OGATA F., 2015, " Nouvelles pratiques funéraires japonaises : de la tombe-ordinateur à la tombe-online ", dans F. DUTEIL-OGATA, I. JONVEAUX, L. KUCZYNSKI et 
S. NIZARD (dir), Le religieux sur internet, Paris, AFSR, L'Harmattan, pp.231-246.

DUTEIL-OGATA F., I. JONVEAUX, L. KUCZYNSKI et S. NIZARD, $2015 \mathrm{a}$, " Introduction », Le religieux sur Internet / Religion on the Web (dossier), dans Online-Heidelberg Journal of Religions on the Internet, $\mathrm{n}^{\circ} 8$, <http://journals.ub.uniheidelberg.de/index.php/religions/issue/view/2132/showToc >, dernière consultation le 2 novembre 2015.

DUTEIL-OGATA F., I. JONVEAUX, L. KUCZYNSKI et S. NIZARD, 2015 b, « Introduction, Le religieux sur internet : textes et contextes ", dans Le religieux sur internet, Paris, AFSR, L'Harmattan, pp.10-27.

DUTEIL-OGATA F., I. JONVEAUX, L. KUCZYNSKI et S. NIZARD (dir), $2015 \mathrm{c}$, Le Religieux sur internet, Paris, Association française de sciences sociales des religions (AFSR), L'Harmattan.

FILIMON-BENEA A., 2014, « Religion et Internet, la tradition à l'épreuve des nouvelles pratiques ", dans S. BRATOSIN, M. A. TUDOR (dir), 2014, Espace public et communication de la foi, s.l. [Les Arcs-sur-Argens], larsic, pp.375-381.

GILLESPIE M., D.E.J. HERBERT, A. GREENHILL (dir), 2013, Social Media and religious change, Berlin, Boston, De Gruyter.

GOLAN O., 2011, « Reconstruire les frontières religieuses en ligne : l'émergence d'un internet juif confessionnel », Le temps des médias, $\mathrm{n}^{\circ} 17, \quad$ pp.118-120, <http://www.histoiredesmedias.com/IMG/pdf/Golan.pdf>, dernière consultation le 2 novembre 2015.

GOMEZ-MEJIA G., 2014, «Un culte hagiographique au sein d'un livre de visages : l'exemple de sainte Rita sur Facebook », MEl, Médiation \& Information, $\mathrm{n} ³ 8, \mathrm{pp} .113-126$.

GONZALEZ P., 2013, Que ton règne vienne, Des évangéliques tentés par le pouvoir absolu, Genève, Labor et Fides.

GRIEVE G. 2010, «Virtually Embodying the Field: Silent Online Buddhist Meditation, Immersion, and the Cardean Ethnographic Method », Online-Heidelberg Journal of Religions on the Internet, vol.4, $\mathrm{n}{ }^{\circ}$.

HADDEN J. K., D. E. COWAN (dir), 2000, Religion on the Internet: research prospects and promises, Bingley (UK), Emerald. 
HELLAND C., 2015, Virtual Religion : A Case Study of Virtual Tibet. Oxford Handbooks Online. New York, Oxford University Press.

HELLAND C., 2014, «Virtual Tibet: Maintaining Identity through Internet Networks », dans Gregory Grieve and Danielle Veidlinger (Eds.), The Pixel in the Lotus: Buddhism, the Internet, and Digital Media, New York, Routledge.

HELLAND C., 2010, « (Virtually) been there, (Virtually) done that: Examining the Online Religious Practices of the Hindu Tradition: Introduction ", Online-Heidelberg Journal of Religions on the Internet, vol.4, $\mathrm{n}^{\circ} 1$.

HELLAND C., 2005, «Online Religion as Lived Religion. Methodological Issues in the Study of Religious Participation on the Internet », Online - Heidelberg Journal of Religions on the Internet, vol.1, $\mathrm{n}^{\circ} 1$.

HOOVER S. M., 2006, Religion in the Media Age, Oxon, Routledge.

HOPE CHEONG P., P. FISCHER-NIELSEN, S. GELFGREN, C. ESS (dir), 2012, Digital Religion, Social Media and Culture, Bern, Peter Lang.

HOWARD R. G., 2011, Digital Jesus, The Making of a New Christian Fundamentalist Community on the Internet, New York et Londres, New York University Press.

JONVEAUX I., 2015, «La virtualité comme catégorie religieuse? Continuité et discontinuité des monastères catholiques en ligne et hors ligne ", dans F. DUTEIL-OGATA, I. JONVEAUX, L. KUCZYNSKI et S. NIZARD (dir), Le religieux sur internet, Paris, AFSR, L'Harmattan, pp.65-79.

JONVEAUX I., 2013, Dieu en ligne, Expériences et pratiques religieuses sur Internet, Paris, Bayard.

JONVEAUX I., 2007, «Une retraite de Carême sur Internet », Archives de Sciences Sociales des Religions, $\mathrm{n}^{\circ}{ }^{\circ} 39$, pp.157-176.

JONVEAUX I., 2009, « L'autre Internet: les moines et le web », Terrain et travaux, $\mathrm{n}^{\circ} 15, \mathrm{pp} .29-50$.

JULLIARD A., 2015, «La mission d'internationaliser St Nicolas, regards ethnologiques sur le site Stnicholascenter.org de culture religieuse anglicane ", dans $\mathrm{F}$. DUTEIL-OGATA, I. JONVEAUX, L. KUCZYNSKI et S. NIZARD (dir), Le religieux sur internet, Paris, AFSR, L'Harmattan, pp.127-139. 
KARAFLOGKA A., 2006, e-religion, A critical appraisal of religious discourse on the world wide web, Londres, Equinox.

KIRSCHLEGER P.-Y., 2015, «Aux commandes du premier réseau protestant francophone, Jesus.net », dans F. DUTEILOGATA, I. JONVEAUX, L. KUCZYNSKI et S. NIZARD (dir), Le religieux sur internet, Paris, AFSR, L'Harmattan, pp.157-171.

KRÜGER O., 2005, «Methods and Theory for Studying Religion on the Internet: Introduction to the Special Issue on Theory and Methodology ", Online-Heidelberg Journal of Religions on the Internet, vol.1, $\mathrm{n}^{\circ 1}$. Fayard.

LAGRÉE M., 2000, La Bénédiction de Prométhée, Paris,

LAMBERT F. (dir), 2014, Prières et propagandes, études sur la prière dans les arènes publiques, suivi du livre I de La Prière de Marcel Mauss, Paris, Hermann.

LAMBERT F., 2013, Je sais bien mais quand même : essai pour une sémiotique des images et de la croyance, Paris, Non standard.

LATOUR B., 2012, Enquête sur les modes d'existence. Une anthropologie des Modernes, Paris, La Découverte.

LÖVHEIM M., 2015, « Une voix à elles : jeunes, musulmanes et blogueuses ", dans F. DUTEIL-OGATA, I. JONVEAUX, L. KUCZYNSKI et S. NIZARD (dir), Le religieux sur internet, Paris, AFSR, L'Harmattan, pp.299-320.

MAYER J.-F., 2008, Internet et religion, Gollion (Suisse), Infolio, Religioscope.

MOTTIER D., 2015, « Le télé-fidèle existe-t-il ? Enquête au sein d'une église pentecôtiste de la région parisienne », dans F. DUTEIL-OGATA, I. JONVEAUX, L. KUCZYNSKI et S. NIZARD (dir), Le religieux sur internet, Paris, AFSR, L'Harmattan, pp.173-187.

NICULESCU M., 2015, «Se connecter et prendre l'avion. Les réseaux électroniques et physiques de la Jewish Mindfulness », dans F. DUTEIL-OGATA, I. JONVEAUX, L. KUCZYNSKI et S. NIZARD (dir), Le religieux sur internet, Paris, AFSR, L'Harmattan, pp.140-155.

PIETTE A., 2003, Le fait religieux, une théorie de la religion ordinaire, Paris, Economica.

PODSELVER L., 2015, “De la visibilité à la présence virtuelle. Quelques fragments de l'expression du judaïsme sur le Net », dans F. DUTEIL-OGATA, I. JONVEAUX, 
L. KUCZYNSKI et S. NIZARD (dir), Le religieux sur internet, Paris, AFSR, L'Harmattan, pp.289-297.

ROY O., 2000, "La communauté virtuelle. L'internet et la déterritorialisation de l'islam ", Réseaux, vol.18, n`99, pp.219237 , $<$ http://www.persee.fr/doc/reso_07517971_2000_num_18_99_2201>, dernière consultation $\mathrm{le} 1^{\text {er }}$ novembre 2015.

SALATKO, Gaspard, 2014, "Décrire le sanctuaire comme opérateur de mise en présence du divin », MEl, Médiation \& Information, $\mathrm{n}^{\circ 38}$, pp.153-165.

SCHEIFINGER H., 2010, "Hindu Embodiment and the Internet », Online-Heidelberg Journal of Religions on the Internet, vol.4, $\mathrm{n}^{\circ}$.

SMITH J. Z., 2014, Magie de la comparaison et autres études d'histoire des religions, choix et trad. D. Barbu et N. Meylan, Genève, Labor et Fides.

SOUZA AGUIAR C. E., 2014, «Les limites de la communication numérique de la foi catholique ", dans S. BRATOSIN et M. A. TUDOR (dir), 2014, Espace public et communication de la foi, s.l. [Les Arcs-sur-Argens], Iarsic, pp.353-364.

STOLOW J., 2014, "Religion et communication: du judaïsme orthodoxe au spiritualisme technique " (entretien avec D. Douyère), MEI, Médiation \& Information, n³8, pp.2142.

STOLOW J. (dir), 2012, Deus in Machina: Religion, Technology, and the Things in Between, New York, Fordham University Press.

VANEL C., 2015, « Mormonisme et internet, entre promotion institutionnelle et régulation de l'utilisation individuelle ", dans F. DUTEIL-OGATA, I. JONVEAUX, L. KUCZYNSKI et S. NIZARD (dir), Le religieux sur internet, Paris, AFSR, L'Harmattan, pp.275-288.

VARLIK S., 2015, "Le discours islamique sur internet : oralité de l'écriture et fragmentation de l'oralité ", dans F. DUTEIL-OGATA, I. JONVEAUX, L. KUCZYNSKI et S. NIZARD (dir), Le religieux sur internet, Paris, AFSR, L'Harmattan, pp.49-63.

VEKEMANS T., 2014, "Double-clicking the Temple Bell. Devotional aspects of Jainism online ", Online-Heidelberg Journal of Religions on the Internet, $\mathrm{n}{ }^{\circ} \mathrm{G}$. 
De la mobilisation de la communication numérique

par les religions

WHEELER, K. R., 2014, «Remixing Images of Islam. The Creation of New Muslim Women Subjectivities on YouTube », Online - Heidelberg Journal of Religions on the Internet, $\mathrm{n}{ }^{\circ} 6$.

ZÉNOUDA H., F. RENUCCI (2014), «Détournement du langage et industrialisation du sacré : l'exemple du mouvement "la Kabbale" ", MEl, Médiation \& Information, n³8, pp.127139. 